\title{
Optical Properties of Nanostructure in CdS at Different Condition Bath Deposition
}

\author{
Ali M. Mousa, Adawiya Jamah Haider and Selma M.Hassan Al-Jawad \\ Department of Applied Sciences, University of Technology, Baghdad, Iraq
}

Received: June 11, 2010 / Accepted: July 09, 2010 / Published: February 10, 2011.

\begin{abstract}
In this work, CdS films were prepared by using chemical bath deposition. Many growth parameters have been considered in this work to specify the optimum condition, namely temperature of solution, $\mathrm{pH}$, thiourea ion concentration and deposition time. Optical properties of CdS films are investigated and analyzed extensively with respect to growth conditions. Nanocrystalline CdS have been deposited, the band gap changes from $2.58 \mathrm{eV}$ to $2.8 \mathrm{eV}$ as compared to the bulk $\mathrm{CdS}$ band gap of $2.42 \mathrm{eV}$, as a result of quantum confinement.
\end{abstract}

Key words: Nanostructure, chemical bath deposition, optical properties.

\section{Introduction}

Nanocrystalline semiconductor particles or quantum dots (QDs) are tiny spheres (10-100 angstrom diameter) of conventional semiconductor, such as $\mathrm{CdS}, \mathrm{ZnS}, \mathrm{PbS}$, CdSe [1-3]. They exhibit physical and electronic properties very different from that observed in the bulk solid semiconductor. These differences arise primarily from quantum confinement effects, the reduction of band structure into discrete quantum levels resulting from the limited size of the particle. The advent of reliable production and characterization of nanostructures has opened a frontier in the development and application of new materials and devices. These nanostructures have interesting physical properties due to their small size and they are often perfect crystals free of defects or internal strain $[4,5]$. Most studied nanocrystalline semiconductors belong to the II-VI group as they are relatively easy to synthesize and are generally prepared as particulates or in thin film form. Among II-VI compounds, CdS is one of the most studied materials. There are different ways to

Corresponding author: Adawiya Jamah Haider (1974- ), female, research fields: laser and semiconductor. E-mail: Adawiya haider@yahoo.com. synthesize CdS nanoparticles such as colloidal particulates, chemical deposition, sol-gel, magnetron sputtering, etc.. Chemical bath deposition (CBD) is the cheapest method in preparing semiconductor films. It allows one to control the deposition process on different substrates with good stoichiometry [6-9]. It is known that nanocrystalline semiconductors (a few $\mathrm{nm}$ ) possess physical properties that are intermediate between those corresponding to the bulk solid and molecules. Depending upon the crystalline size, two limiting confinements can be observed in practice [10, 11]. When the radius of the crystallite $R$ is smaller than $\sim 2$ exciton Bohr radii, electrons and holes are considered as two confined particles, bound by an enforced Coulomb interaction, and when the crystallite radius is larger than exciton radii, the ground exciton is treated as a rigid sphere, confined as a quasiparticle. Between these two limiting cases both the electron and hole confinement and their Coulomb interaction are considered. In case of nanocrystallites, the electrons, holes and excitons have limited space to move and their motion is possible for definite values of the energy. As a result, the continuum of states in the conduction and valence band are broken down into discrete states with 
an energy spacing, relative to the band edge, which is approximately inversely proportional to the square of the particle radius resulting in widening of the band gap compared to the bulk. This is normally observed by a blue shift in the optical absorption spectra [12,13]. We try to investigate the influence of the different deposition condition on quantum size effects.

\section{Experimental Procedures}

CdS films were grown using CBD technology on glass slides. This technology offers the deposition of a thin uniform film with a minimal thickness on the substrate surface. Substrate used for deposition CdS is borosilicate glass slides, which were first cleaned in distilled water in order to remove the impurities and residuls from their surfaces. Followed it rinsing in chromic acid (for one day), to introducing functional groups called nucleation and/or epitaxial centers, which formed the basis for the thin films growth. Then, repeated washing in deionized water, and finally put in ultrasonic wave with distilled water for $15 \mathrm{~min}$ then dried.

The reaction cell was a $100 \mathrm{~mL}$ beaker containing $0.1 \mathrm{M} \mathrm{CdSO}_{4}$ aqueous solution, $0.2 \mathrm{M}$ thiourea aqueous solution and 5.6 $\mathrm{M} \mathrm{NH}_{4} \mathrm{OH}$ in the volume ratio 1: 1: 1 . The substrate was then suspended in the reaction cell. Reasonably good films of CdS were obtaind in $20 \mathrm{~min}$ at a constant temperature. The freshly grown films were thoroughly washed by water jet, dried and kept in a vacuum. The different crystalline sizes were obtained by controlling the reaction time.

The crystal structure of the films was determined by XRD (using $\mathrm{CuK} \alpha$ radiations, $\lambda=1.54 \mathrm{~A}$ ). The film thickness was measured by optical interferometer method. Optical transmission spectra was taken by a Cecile CE 7200 Spectrophotometer supplied by Aquarius company for CdS/glass thin films.

\section{Results and Discussion}

3.1 Structure Properties of Nano-Structure CdS
Fig. 1 shows the XRD spectra from the CdS films deposited onto glass substrate. According to the X-ray diffraction results, the spectra reveal that deposited films are polycrystalline in nature. By comparing with ASTM, the XRD pattern of the deposited films exhibits peaks at $\left(2 \theta=28^{\circ}\right)$ with the $\mathrm{H}(100)$ is the most intense and resolved peak. No peaks corresponding to elemental $\mathrm{Cd}$ which could be attributed to the strongly bounded $\mathrm{Cd}$ in the formation of CdS compound. This could be explained by adopting the approach of S. Ram and C. Mitra (In controlled chemical reaction with reaction species dispersed ion a diluted medium as in the present work), phase with small critical volume both nucleation and growth are easily if compared with that of a bigger critical volume i.e., increasing deposition time accompanied by redistribution entropy in favor of CdS planes. It is also noted that signals from the glass substrate will complicated the XRD spectrum, making it difficult to evaluate average grain size.

The average grain size is calculated by using the Scherrer formula and neglecting peak broadening due to residual stresses in the film $\mathrm{D}=0.9 \lambda / \beta \cos \theta$ where $\lambda$ $=1.54 \mathrm{~A}^{\circ}$ for $\mathrm{CuK} \alpha, \beta$ is the full width at half maximum of the peak and $\theta$ is the Bragg angle. The lattice parameter (a) of the films was calculated using the relation $d=a /\left(h^{2}+k^{2}+l^{2}\right)^{0.5}$ where $h, k$ and 1 are miller indices [14].

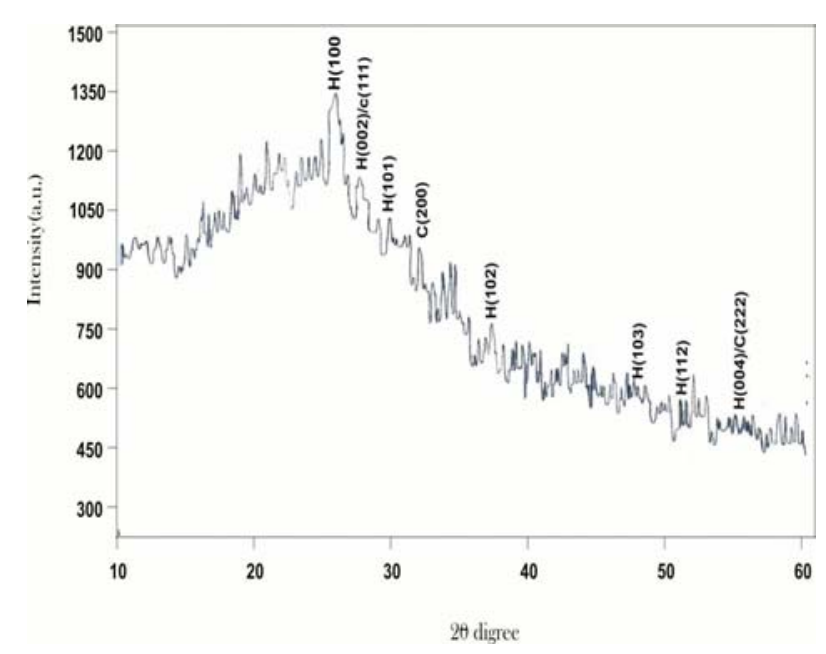

Fig. 1 X-ray diffraction of nano CdS films. 


\subsection{Transmission}

Optical transmission spectra depends on the chemical and crystal structure of the films, and also on the film thickness and on films surface morphology. The effect of different deposition parameters on these spectra is shown in Fig. 2. It has been found that the films have high transmission at long wave lengths approximately (70-80\%), and decreasing transmission to $(10 \%)$ at short wave lengths.

Films transmission decreases with increasing deposition time and temperature at wave lengths higher than the absorption edge. On the other hand, increasing thiourea ion concentration increases transmission. These opposite effects could be attributed to the increased abundance and/or size of the single crystallite overgrowth. Fig. 2 shows the effect of $\mathrm{pH}$ on the optical transmission spectra of the as-deposited CdS films. It was observed that the transmission of the film decreases with increasing in $\mathrm{pH}$ up to 11.5 after

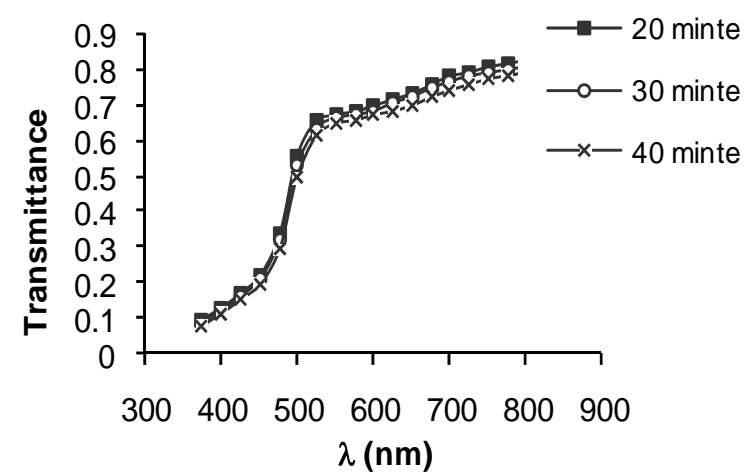

(a)

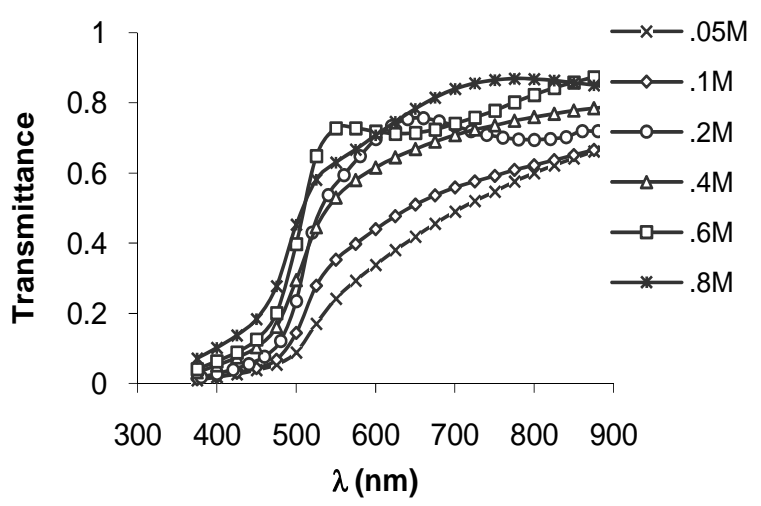

(c) which it starts increasing with further increase in $\mathrm{pH}$. The increase and decrease in the transmittance percentage during the continuous increase in $\mathrm{pH}$ is due to the transition of $\mathrm{CdS}$ phase from mixed cubic and hexagonal to hexagonal structure, and also due to change in the films thickness with the $\mathrm{pH}$ of the solution. With this result we think that $\mathrm{pH} 11.5$ is the optimum value to deposit $\mathrm{CdS}$ film for photovoltaic application.

\subsection{Absorption Coefficient}

The data from transmission spectrum are used to calculate absorption coefficient. Fig. 3 shows the optical absorption spectra recorded for the CdS films for different deposition parameters. It is clear that the films have high absorption coefficient at short wave length range $(375-500 \mathrm{~nm})$, then decrease at different rates dependence on the films structure to reach constant values at long wave lengths which it above from the

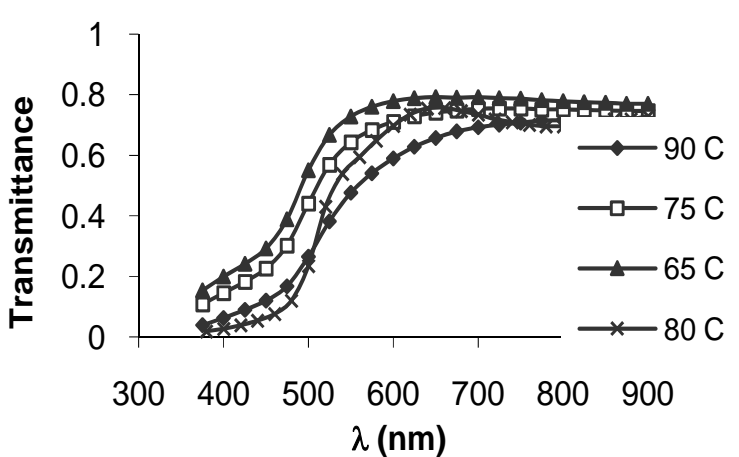

(b)

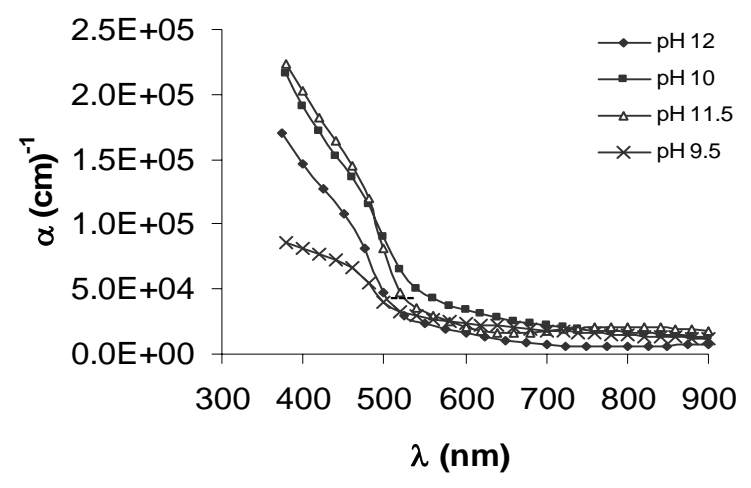

(d)

Fig. 2 The optical transmission spectra of CdS films for different deposition parameters: (a) For different times, (b) For different temperatures, (c) For different thiourea ion concentrations, (d) For different pH. 


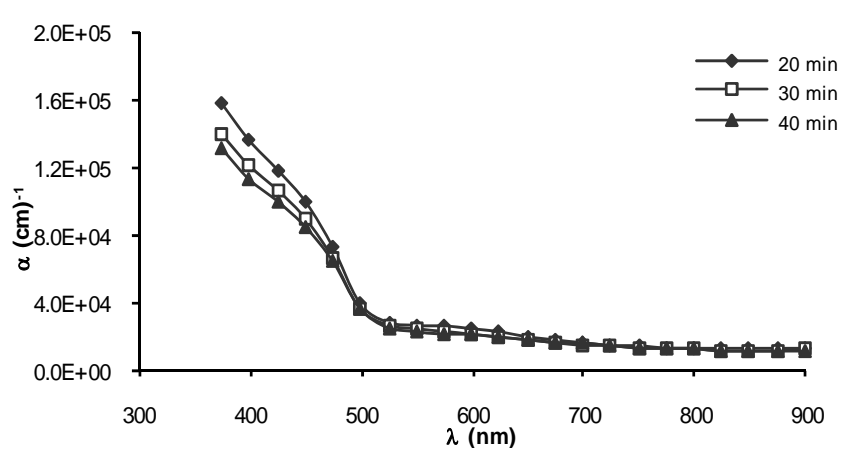

(a)

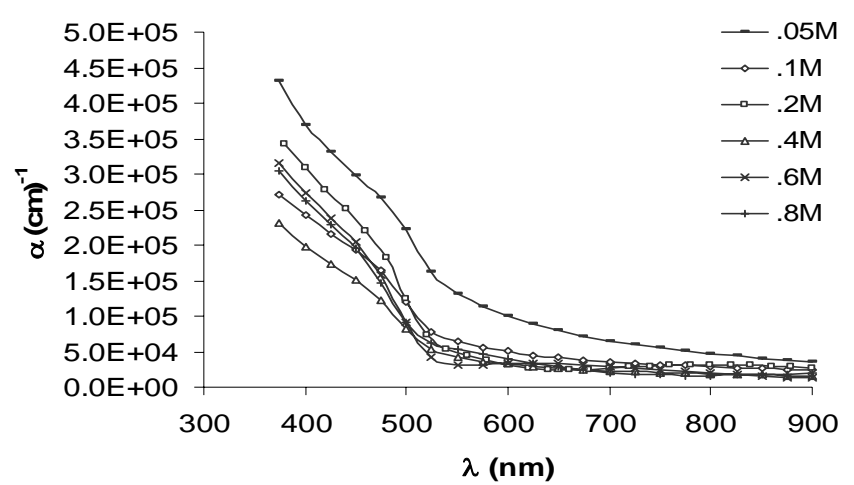

(c)

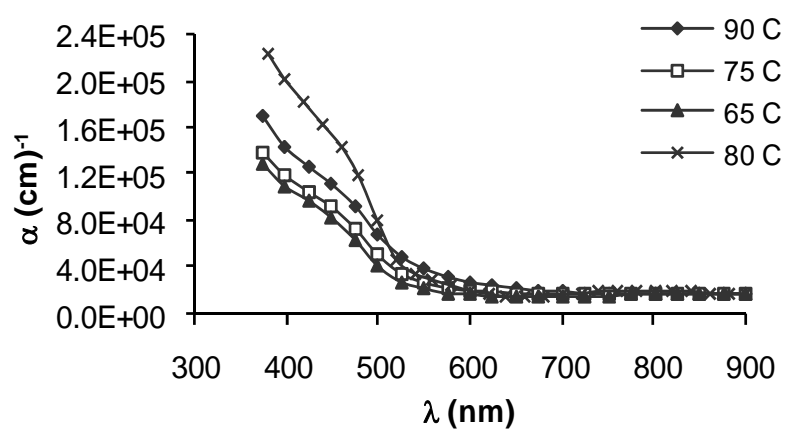

(b)

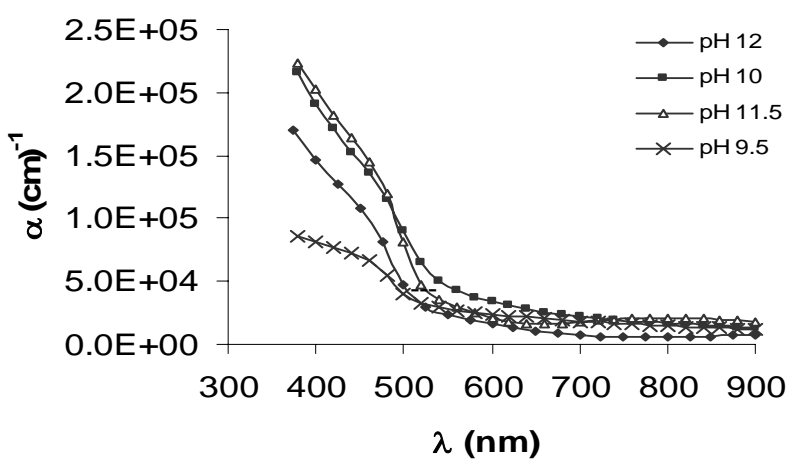

(d)

Fig. 3 The optical absorption spectra for the CdS films for different deposition parameters: (a) For different times, (b) For different temperatures, (c) For different thiourea ion concentrations, (d) For different pH.

$520 \mathrm{~nm}$, where the films become transparence at this wave lengths. The approximately same fundamental absorption edge is observed at about $520 \mathrm{~nm}$ for all the samples with some difference. The figure shows results of the films prepared at high temperature, and low thiourea ion concentration have high optical absorption coefficient, where the value reaches more than $\left(1.8 \times 10^{5} \mathrm{~cm}^{-1}\right)$.

\subsection{Optical Energy Gap}

The energy gap values depends in general on the films crystal structure, the arrangement and distribution of atoms in the crystal lattice, also it is affected by crystal regularity. The energy gap (Eg) value is calculated by extrapolation of the straight line of the plot of $(\alpha h v)^{2}$ versus photon energy for different deposition parameters as shown in Fig. 4. The linear dependence of $(\alpha h v)^{2}$ with (hv) indicates direct band gap. The band gap increases with the increase in thiourea ion concentration. More probably this is related to the decrease in grain size with increasing deposition rate. The deposition rate influences the c-lattice constant, which is determined by the amount of strain at the substrate larger interface [15]. The band gap changes with the $\mathrm{pH}$ of the solution at constant thiourea ion concentration. This also can be attributed to the decrease in grain size with increase in deposition rate. The band gap values for different deposition parameters are tabulated in Table 1.

\subsection{Effect of Nanocrystalline}

The results of optical transmittance spectrum of the CdS films prepared for different times are presented in Fig. 5. All films exhibit a high transmittance of about $90 \%$ in the visible region which falls sharply in the short wave length. The fairly sharp cutoff obtained over a particular wavelength range suggests that one particle size is dominant. The sharpness of the curve near maximum transmission varies from one sample to another suggesting variation in particle size distribution from one sample to another [16].

Fig. 6 shows the absorption spectra of different short 


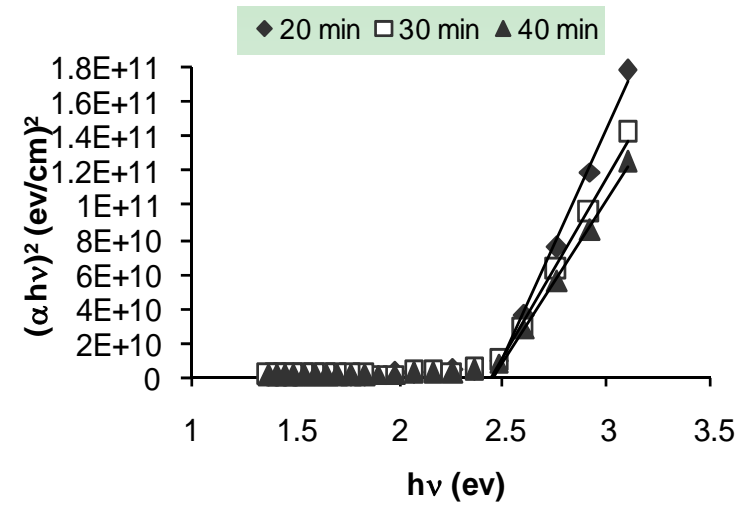

(a)

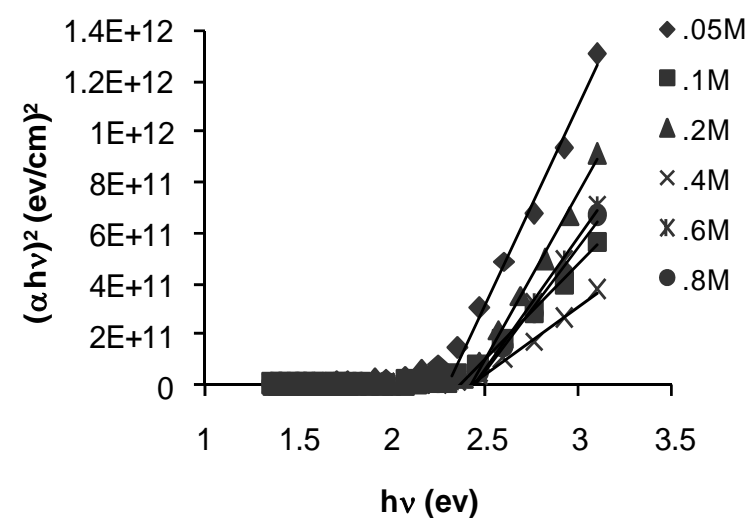

(c)

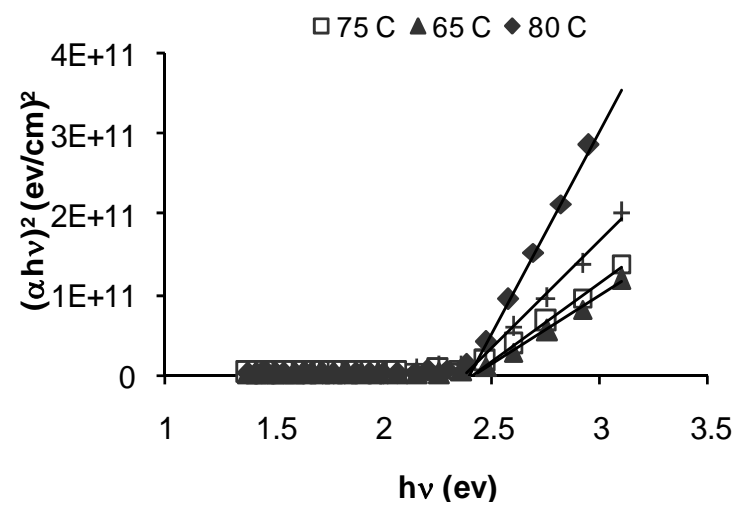

(b)

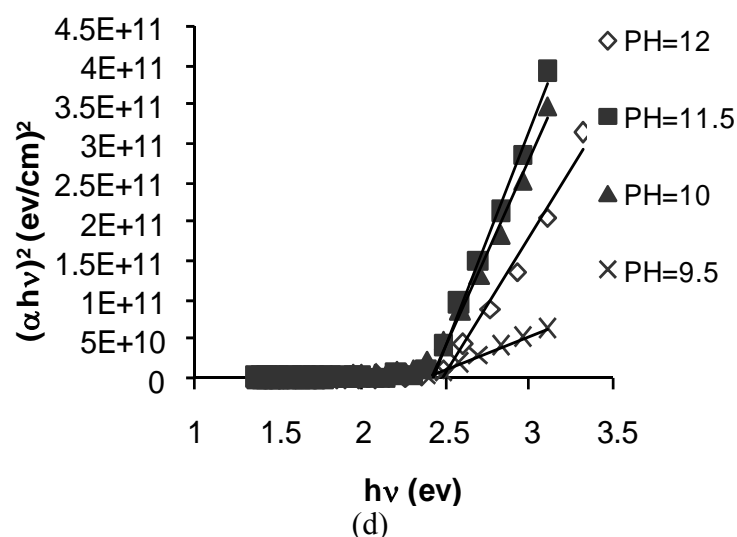

(d)

Fig. 4 A plots of $(\alpha h v)^{2}$ verses (hv) of CdS thin films for different deposition parameters.: (a) For different times, (b) For different temperatures, (c) For different thiourea ion concentrations, (d) For different pH.

Table 1 Experimental results.

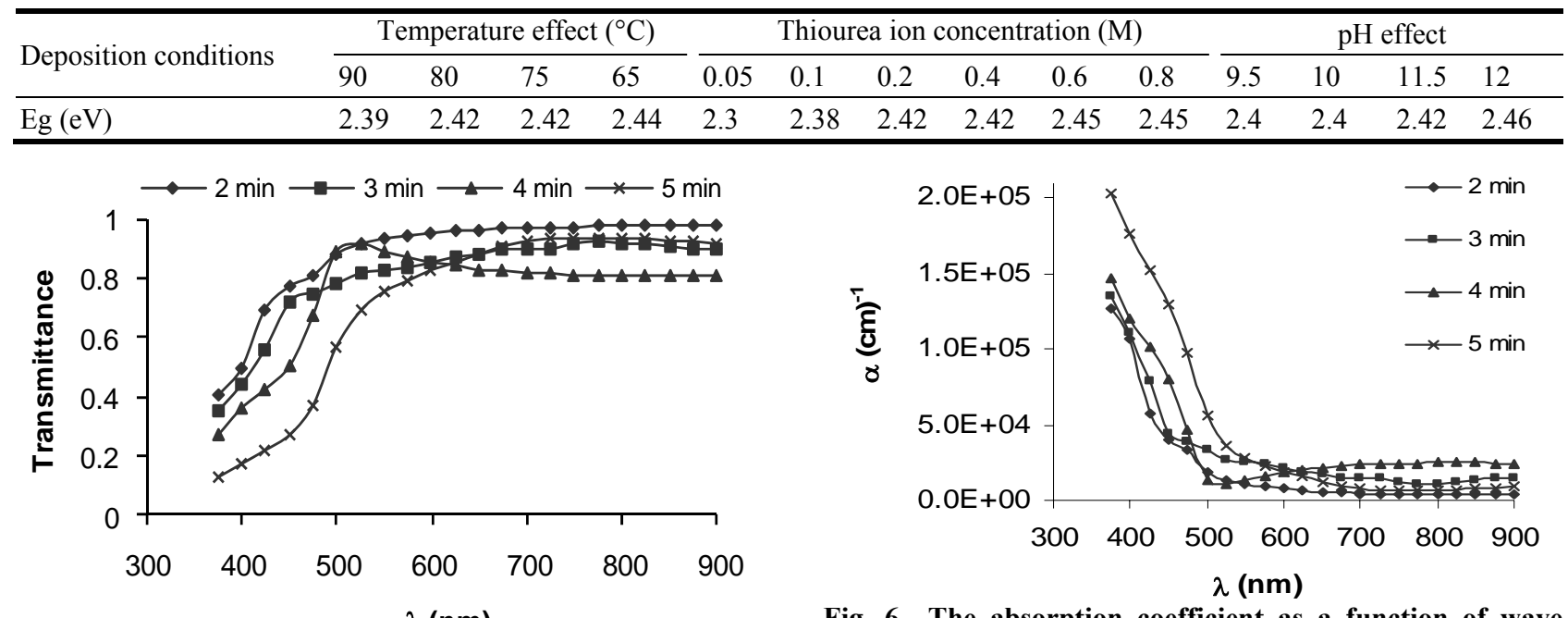

Fig. 5 The optical transmission spectra of nanocrystalline CdS films.

deposition times. As the crystal size decreases, there is a blue shift of the absorption edge which is a signature of the size quantization effect. These results are in good
Fig. 6 The absorption coefficient as a function of wave length for nanocrystalline CdS films.

agreement with results of K.K. Nanda et al. [10].

As CdS is a direct band gap semiconductor, the band gap is estimated from the plots of $(\alpha h v)^{2}$ versus (hv) and shown in Fig. 7, and it has a change in the band gap 
from $2.42 \mathrm{eV}$ (bulk) to $2.85 \mathrm{eV}$ (nanocrystalline). According to the effective mass approximation, one can use the energy position to estimate the average grain size. The grain size dependence on the band gap is shown in Fig. 8. Also Table 2 contains the results of energy gap and average grain size as a function of time, and these results agree with many studies.

The basic principle for $\mathrm{CBD}$ of $\mathrm{CdS}$ requires the presence of both constituent ions i.e., $\mathrm{Cd}^{2+}$ and $\mathrm{S}^{2-}$. The sulfide ions can be produced by the hydrolysis of the thiourea in a basic medium with $\mathrm{pH}=10-12$ or in aqueous $\mathrm{NH}_{3}$ [17], while the cadmium ions can be supplied by dissolved cadmium salts like $\left(\mathrm{CdSO}_{4}\right)$ [18-20].

\section{Conclusions}

Cadmium sulfide deposited by CBD at low deposition time (less than $5 \mathrm{~min}$ ) is fond to be nanocrystalline, the

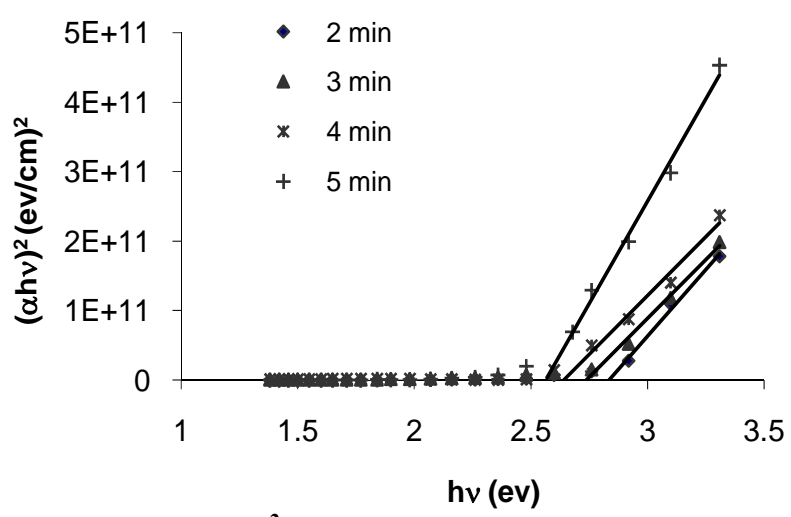

Fig. 7 Plot of $(\alpha h v)^{2}$ versus (hv) for nanocrystalline CdS.

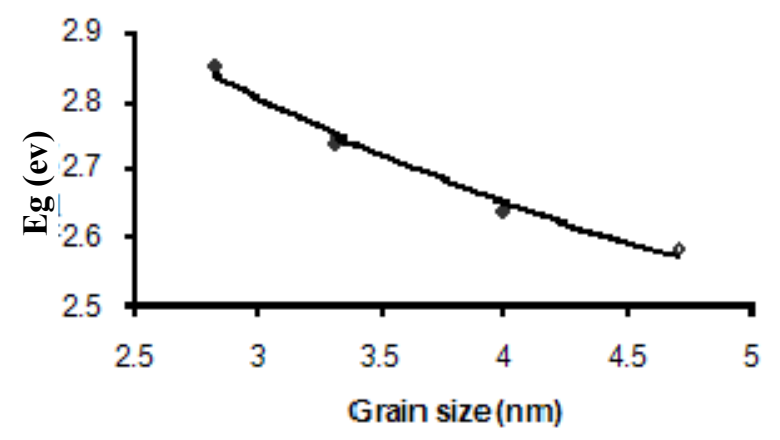

Fig. 8 Relationship between grain size and energy gap.

Table 2 The results of energy gap and average grain size as a function of time.

\begin{tabular}{lllll}
\hline Time $(\mathrm{min})$ & 2 & 3 & 4 & 5 \\
\hline $\mathrm{Eg}(\mathrm{eV})$ & 2.85 & 2.74 & 2.64 & 2.58 \\
\hline
\end{tabular}

crystallite sizes were within $2.85-4.7 \mathrm{~nm}$, and the crystallites increase in size as the deposition time of the $\mathrm{CdS}$ increases. The increase of the grain size with deposition time is probably due to this lateral growth mode. Sharp cutoff obtained over a particular wavelength range suggests that one particle size is dominant. The sharpness varies from one sample to another suggesting variation in particle size distribution.

\section{References}

[1] K.K. Nanda, S.N. Sahu, Study of nanocrystallites by AFM and raman scattering, Applied Surface Science 119 (1997) 50-54.

[2] T. Pisarkiewicz, E. Schabowska, E. Kusior, Cadmium sulfied thin films manufactured by chemical bath deposition, Journal of Wide BandGap Materials 9 (2001) 127-132.

[3] E. Pentia, L. Pintilie, I. Matei, T. Botila, E. Ozbay, Chemically prepared nanocrystalline $\mathrm{PbS}$ thin films, Journal of Optoelectronics and Advanced Materials 3 (2001) 525-530.

[4] M.J. Seong, O.I. Micic, A.J. Nozik, A. Mascarenhas, Size-dependent raman study of InP quntum dotes, Applied Physics Letters 82 (2003) 185-187.

[5] D. Matsuura, Y. Kanemitsu, T. Kushida, C.W. White, J.D. Budai, A. Maldrum, Structure and morphology of chemical bath deposited CdS films and clusters, Applied Physics Letters 77 (2000) 2289-2291.

[6] G.A. Alozie, F.C. Eze, Chemical bath deposition of CdS thin films and their partial conversion to $\mathrm{CdO}$ on annealing, Global Journal of Pure and Applied Science 9 (2003) 591-599.

[7] F.I. Ezema, P.E. Ugwuoke, Spectroscopic characterization of Chemical bath deposited cadmium sulphide laye, The Pacific Journal of Science and Technology 5 (2003) 33-38.

[8] J.G.V. Luna, A. Zehe, O. Zelaya-Angel, Chemical CdS thin film, Cryst. Res. Techno. 34 (1999) 949-958.

[9] I. Grozdanov, A simple and low-cost technique for electroless deposition of chalcogenide, Semicond. Sci. Technol. 9 (1994) 1234-1241.

[10] K.K. Nanda, S.N. Sarangi, S. Mohanty, S.N. Sahu, Optical properties of $\mathrm{CdS}$ nanocrystalline films prepared by precipitation technique, Thin Solid Films 322 (1998) 21-27.

[11] H. Zhang, X. Ma, J. Xu, J. Niu, J. Sha, D. Yang, Deposition and characterization of CdS and CdS:As thin films, J. Crystal Growth 246 (2002) 108-112. 
[12] B.K. Patel, K.K. Nanda, S.N. Sahu, Some properties of thin films of chemically deposited cadmium sulphide, J. Appl. Phys. 85 (1999) 3666-3670.

[13] W. Liu, C. Jin, C. Jia, L. Yao, W. Cai, X. Li, Doping of chemically deposited intrinsic CdS thin films to $\mathrm{n}$ type by thermal diffusion of indium, Chemistry Letters 33 (2004) 228-229.

[14] E.S. Tüzemen, S. Eker, H. Kavak, R. Esen, Dependence of film thickness on the structural and optical properties of ZnO thin films, Appl. Sur. Sci. (2009). (in press)

[15] M.B.O. Lopez, M.S. Lerma, A.M. Galvan, R.R. Bon, Novel approach to the deposition of CdS by chemical bath deposition: The deposition of crystalline thin films of CdS from acidic bath, Thin Solid Films 457 (2004) 278-284.

[16] E.I. Ugwu, D.U. Onah, Influence of $\mathrm{pH}$ values on chemical bath deposited $\mathrm{FeS}_{2}$ thin films, The Pacific Journal of Science and Technology 8 (2007) 1.

[17] P.K. Nair, M.T.S. Nair, Prospects of chemically deposited CdS thin films in solar cell applications, Solar Cells 22 (1987) 103-112.

[18] K.U. Isah, N. Hariharan, A. Oberafo, Leonardo influence of $\mathrm{pH}$ values on chemical bath deposited $\mathrm{FeS}_{2}$ thin films, Journal of Sciences 12 (2008) 111-120.

[19] R.C. Kainthla, D.K. Pandya, K.L. Chopra, Growth kinetic and polymorphism ofchemically deposited CdS films, J. Electrochem. Soc. 127 (1980) 277-283.

[20] G. Hodes, A. Yaron, F. Decker, P. Motisuke, Three-dimensional quntum-size effect in chemically deposited cadmium selenide films, Physical Review B 36 (1987) 4215-4221. 